\title{
First-order theory for Earth's inner-core anisotropy due to super-rotation and Ramachandran interaction
}

\author{
Andrew Das Arulsamy \\ Condensed Matter Group, Institute of Interdisciplinary Science, No. 24, Level-4, Block C, Lorong Bahagia, \\ Pandamaran, 42000 Port Klang, Selangor DE, Malaysia. \\ e-mail: sadwerdna@gmail.com
}

Solidification mechanism at the Lehmann (inner core) boundary are postulated on the basis of Ramachandran interaction by taking the fluctuating inner core super-rotation into account. The postulates are found to be consistent with compressional or P-wave velocity obtained from seismic data analysis. We justify these postulates to be physically sound and precise, and show that the fluctuating inner core super-rotation causes significant changes to the strength of $\mathrm{Fe}-\mathrm{Fe}$ Ramachandran interaction, which then leads to the observed asymmetric and anisotropic inner core. Our postulates also reliably explain that the depth-dependent anisotropic P-wave attenuation close to inner core surface (to about $100 \mathrm{~km}$ deep) is due to phonon excitation probability and different atomic orientation. We also discuss the consistency of our postulates with respect to asymmetric inner core anisotropy (between western and eastern inner core hemispheres).

\section{Introduction}

Oldham, Jeffreys, Mohorovičić, Gutenberg, Lehmann, Richter, Birch and Bullen are the pioneers responsible to help devise the basic Earth model with respect to density, compositional and structural anisotropies along the radial depth of Earth's internal structure, including liquid (outer-core) and solid inner-core (Oldham 1906; Gutenberg 1913; Jeffreys 1926; Gutenberg and Richter 1936; Lehmann 1936; Birch 1964; Bullen 1985; McLeish 1992). This picture is more or less settled with respect to seismic data analysis (Kenneth and Engdahl 1991). In those analyses, PKP seismograms do not give rise to any observable $\mathrm{P}$-wave velocity transition within inner core in order to surmise the existence of a georeactor (composed of radioactive material (alloyed with iron) and its waste) at the center of inner core. Therefore, we will not discuss the possibility of a georeactor-core (Herndon 1992) as already critically reviewed by Rao (2002) and Sankaran (2002).

However, the discovery of free-to-rotate innercore super-rotation ( $\mathrm{Su}$ and Dziewonski 1995; Creager 1997; Richards 2000; Song 2000) exposes a significant gap in our knowledge about the nature of crystal-structure anisotropy in Earth's solid innercore, which was first put forth by Morelli et al. (1986) and Woodhouse et al. (1986) using the PKP(DF) and normal-mode techniques, respectively. The $\operatorname{PKP}(\mathrm{DF})$ technique was first reported by Poupinet et al. (1983). Here, PKP(DF) wave travels through inner core, while $\operatorname{PKP}(\mathrm{AB})$ and $\mathrm{PKP}(\mathrm{BC})$ waves travel through the upper (far from inner core) and lower (close to inner core) sections of liquid outer core without passing through inner

Keywords. Iron crystallization; asymmetric and anisotropic inner core; fluctuating inner core rotation; Ramachandran interaction theory. 
core. $\mathrm{PKP}(\mathrm{CD})$ also avoids inner core because it is reflected at the Lehmann (inner core) boundary. This super-rotation (inner core rotates slightly faster than mantle, about 0.3 degree $\mathrm{yr}^{-1}$ ) is also found to agree with seismic data obtained from earthquake doublets - the second compressional primary (or sound) wave $(\mathrm{P})$ takes different travelpaths through inner-core in relation to the first one (Song 1997; Tromp 2001). Here, different travel-times imply anisotropic inner core, provided that the outer core and mantle do not significantly contribute to these different travel-times. Interestingly, enough information on Earth's inner core crystal structure can be derived from seismic body waves, namely, by detecting the Pand S-wave travel times, their reflections, refractions, and changes to their phase velocities (Song 1997; Tromp 2001). Here, S-wave refers to secondary shear wave that gets severely attenuated in liquid outer core, and travels slower than P-wave (Song 1997; Tromp 2001). The above information obtained from seismograms is sufficient to pin-point that the inner core has got to be anisotropic.

The above-stated anisotropy turned out to be spatially nonuniform (asymmetrically anisotropic), which is a fact in (mostly iron) inner core, surmised from the measured body-wave velocity anisotropy. For example, body-wave speeds in an anisotropic medium are direction-dependent (Song 1997). On the other hand, Earth's normal modes' anomalous splitting also supports the existence of anisotropic inner core (Masters and Gilbert 1981). However, we will not be able to relate our proposed theory to such normal modes, including the simplest modes, namely, the spheroidal $\left({ }_{0} S_{2}\right)$ and toroidal $\left({ }_{0} T_{2}\right)$ modes. In any case, the current state of affairs is that the solidification mechanism needed to explain the existence of both asymmetric and anisotropic inner core crystal structure, and the east-west asymmetric anisotropy (Shearer and Toy 1991; Tanaka and Hamaguchi 1997) remains unknown (Shearer and Toy 1991; Su and Dziewonski 1995; Song 1997; Tanaka and Hamaguchi 1997; Tromp 2001; Mattesini et al. 2013; Tkalčić et al. 2013). We stress that this last issue is the most difficult one and needs to be handled properly and consistently. Unfortunately, we will not be able to find a convincing argument to explain the tilted cylindrical symmetry (tilted from the Earth's spin axis: $79.5 \pm 1.0^{\circ} \mathrm{N}$ and $\left.160 \pm 5^{\circ} \mathrm{E}\right)(\mathrm{Su}$ and Dziewonski 1995). A proper explanation is possible however, if one could check the consistency of this tilting by analyzing the PKP(DF) data on both sides of Earth's spin axis (from both eastern and western hemispheres), and the angles made by PKP(DF) waves before entering, and after exiting the inner core.
In any case, our primary task here is to first construct the postulates that can explain the solidification mechanism microscopically on the basis of $\mathrm{Fe}-\mathrm{Fe}$ Ramachandran interaction (Arulsamy 2013, 2014a) at the Lehmann interface by taking the fluctuating inner core super-rotation $(\mathrm{Su}$ and Dziewonski 1995; Tkalčić et al. 2013) into account. Note that, crystallization is a complex process in general, and therefore, has not been properly captured in terms of microscopic physics (Desiraju 2007), hence we start by building proper postulates. The Ramachandran interaction exists between two atomic Fe, one from outer core and another from inner core. Subsequently, we shall see whether the theory can give unequivocal explanations on the solidification mechanism that give rise to anisotropic inner core obeying the wellestablished P-wave velocities obtained from seismic data analysis. Even though the above solidification mechanism is microscopic, it is strictly based on two postulates, which need to be experimentally verified (at least indirectly) by means of a crystal growth technique.

These postulates need to consistently explain the following experimental findings. (i) Directiondependent $\mathrm{P}$-wave velocities passing through different parts of inner core. For example, P-wave traveling close to north-south (polar) path is always faster (about $2 \mathrm{~s}$ or more) than the one passing near the equatorial (west-east) (Poupinet et al. 1983) - the slow equatorial path is deduced from an earthquake in Peru to station KOD in Kodaikanal, Tamil Nadu, India, while the fast polar path is obtained from an earthquake in the South Sandwich Islands region (southern Atlantic Ocean) to COL at College, Alaska, USA (Song and Helmberger 1993). The above slow and fast paths are not determined solely from two earthquakes. In fact, the geometry of the inner core anisotropy has of course been determined (and is still being refined) from many different earthquakes. (ii) The top $500 \mathrm{~km}$ of the western inner core hemisphere (under central America) is non-uniformly anisotropic with slow equatorial $\mathrm{P}$-wave speed, while the eastern hemisphere (under the Indian Ocean) is almost uniformly anisotropic with high equatorial P-wave speed (Shearer and Toy 1991; Tanaka and Hamaguchi 1997). (iii) P-wave is highly attenuated in the top $100 \mathrm{~km}$, and it decreases with depth (Doornbos 1974) and this attenuation is also anisotropic (Souriau and Romanowicz 1996). Finally, we also highlight the possibility to falsify our postulates using a well-known experimental technique to grow hexagonal close-packed (hcp) $\mathrm{Zn}-\mathrm{Cu}$ alloy (about $420 \mathrm{~K}$ and pressure $1 \mathrm{~atm}$ ), or alternatively, one can grow hcp Co metal by introducing fluctuating angular velocity (during crystal growth). 


\section{Theoretical details}

In the following subsections, we first derive the appropriate physics needed to understand the variation of $\mathrm{P}$-wave velocity due to different atomic orientation. We consider the iron alloy to be just below the Lehmann boundary or sufficiently away from the critical point (inner core surface). This is followed by discussion on the physics of Ramachandran attraction between two iron atoms (right at the Lehmann boundary or critical point). One of the iron atoms is from molten iron, and the other from solid inner core surface.

\subsection{P-wave velocity}

To obtain the suitable $\mathrm{P}$-wave phase velocity formula that can be handled analytically even for complex systems, we start from (Hill 1952),

$$
V_{\mathrm{P}}=\sqrt{\frac{K_{\mathrm{b}}+(4 / 3) G_{\mathrm{s}}}{\rho} .}
$$

This velocity is chosen because it is one of the most important experimental quantities extracted from seismic data analysis. For an obvious reason, equation (1) has got nothing to do with group velocity. The velocity defined in equation (1) is a function of inner core density $(\rho)$, its bulk $\left(K_{\mathrm{b}}\right)$ and shear $\left(G_{\mathrm{s}}\right)$ moduli, which can be physically understood in the following way $-V_{\mathrm{P}}^{2} \propto K_{\mathrm{b}}$ and $V_{\mathrm{P}}^{2} \propto G_{\mathrm{s}}$ because highly rigid solid (that has high frequency phonons $\left.\left(\omega_{\mathrm{ph}}\right)\right)$ transports body waves faster due to this relation, $t_{\mathrm{P}} \propto 1 / \omega_{\mathrm{ph}}$ where $t_{\mathrm{P}}$ denotes $\mathrm{P}$ wave travel time. In addition, $V_{\mathrm{P}}^{2} \propto 1 / \rho=V / M$ requires low-mass solid (for fixed volume) to obtain large $V_{\mathrm{P}}$ because highly rigid low-mass $(m)$ solid gives rise to high $\mathrm{P}$-wave velocity as a result of this fact, $\omega_{\mathrm{ph}}=\sqrt{k / m}$, where $V$ and $k$ are the respective volume and interaction potential (also known as the spring) constant between an atomic $\mathrm{Fe}$ and its surrounding sea of free electrons. The mass for atomic Fe in solid phase, $m$ here refers to a particular unit cell such that $M=\sum_{i} m_{i}$.

We now introduce our crucial approximation: $\mathrm{P}$-wave has to travel through the fast and slow axes of all the crystal structure orientations found along the Maupertuis principle-of-least-action path (Emery 1988), and therefore, we can effectively represent the $\mathrm{P}$-wave path with one dimensional atomic arrangements. Without this approximation, the one-dimensional equation of motion in an arbitrary crystal along the edge (say, along the $x$-axis) is given by (Kittel 1957)

$$
\rho \ddot{u}=c_{11} \frac{\partial^{2} u}{\partial x^{2}},
$$

which is nothing but the wave equation in one dimension, and $u$ is the atomic displacement in the $x$ direction. The solution to equation (2) is known as the longitudinal mechanical wave, $u=$ $u_{0} \exp [i(\omega t-k x)]$, which eventually leads us to obtain the required phase velocity (from equation 2)

$$
v_{\mathrm{P}}=\frac{\omega_{\mathrm{ph}}}{k}=\frac{1}{\sqrt{k \rho V}}=\sqrt{\frac{c_{11}}{\rho}},
$$

where $c_{11}$ is the well known elastic stiffness constant (or elastic modulus), and $v_{\mathrm{P}}$ is the $\mathrm{P}$-wave velocity activated by the seismic body waves. Activating our approximation stated above means equation (3) needs to be rewritten to read,

$$
v_{\mathrm{P}}=\sqrt{c^{\mathrm{s}}} \sum_{i} \sqrt{\frac{1}{\rho_{i}}} .
$$

Here, equation (4) is a transformed one-dimensional representation of equation (1) such that the sum takes into account each different set of fast and slow (phase velocity) axes for different atomic orientations and crystal structures. For example, $i=1$ is for one set (of axis and atomic orientation of a crystal structure), while $i=2$ is for another set, and so on, taking into account the possible axes along the Maupertuis least action path taken by a particular P-wave after an earthquake. Most importantly, the newly approximated elastic stiffness constant, $c^{\mathrm{s}}$ can be treated as a three-dimensional constant because $c^{\mathrm{s}}$ is only weakly $\mathrm{Fe}-\mathrm{Fe}$ orientation dependent, at least in iron Fermi metals with metallic bonds such that $\omega_{\mathrm{ph}}=\sqrt{c^{\mathrm{s}}}\left[k_{i} / \sqrt{\rho_{i}}\right]$.

Moreover, we require $c^{\mathrm{s}}$ not proportional to $\rho$ because such a proportionality $\left(c^{\mathrm{s}} \propto \rho\right)$ will lead to a constant $v_{\mathrm{P}}$, and this should not be the case (see the discussion after equation (11) for more details). In addition, $c^{\mathrm{s}}$ being not proportional to $\rho$ is a reasonable statement for Fermi metals with three-dimensionally identical metallic bonds, provided that there is no comparison with other Fermi metals or systems with different applied pressure. In other Fermi metals, there is a possibility for one to have $\omega_{\mathrm{ph}}=[1 / \sqrt{\rho}] \sqrt{c_{i}^{\mathrm{s}}} k_{i}$ such that the effect of $c_{i}^{\mathrm{s}}$ is more pronounced than $\rho_{i}$ along a given crystallographic axis. All these side effects will be made clear after equation (11). For now, we can readily see that $c^{\mathrm{s}} \propto K_{\mathrm{b}}+G_{\mathrm{s}}$, which implies $v_{\mathrm{P}} \propto V_{\mathrm{P}}$. Hence, the subscript $i$ actually counts the number of fast and slow axes found along the Maupertuis least action one-dimensional path travelled by the P-wave. Consequently, the physics explained earlier for equation (1) remains the same for equation (4). 


\subsection{Ramachandran interaction}

To understand the formation of solid iron inner core due to cooling, we need to determine the attraction between an atomic $\mathrm{Fe}$ (from solid inner core) and another atomic Fe (from liquid outer core) that can eventually lead to liquid-tosolid chemical reaction. The stated attraction is due to Ramachandran interaction ( $V_{\text {Ramachandran }}$ ) (Ramachandran et al. 1963), which is defined to exist between non-bonded atoms, which is stronger than the strongest van der Waals (vdW) attraction (for neutral polarizable atoms) (Arulsamy 2014a), including the non-covalent carbon bond (Arunan 2013; Mani and Arunan 2013), the blue-shifting hydrogen bond (for neutral polarizable atoms) (Hermansson 2002), and also between an ion and a polarizable neutral atom (Arulsamy 2014a).

Different atomic orientation for an hcp crystal structure is expected if $V_{\text {Ramachandran }}$ fluctuates with a timescale of the order of super-rotation rate. We shall return to this expected statement in a short while. First, we define $V_{\text {Ramachandran, which is }}$ strong enough to overcome the polarized electronelectron (el-el) repulsion to initiate chemical reaction between Fe atoms, leading to iron crystallization. The relevant Ramachandran attraction operator is given by (derived elsewhere (Arulsamy 2014a)):

$$
V_{\text {Ramachandran }}=V_{\text {Coulomb }}^{\text {e-ion }}+\frac{1}{2} V_{\text {Ramachandran }}^{\mathrm{I}} .
$$

Here, $V_{\text {Coulomb }}^{\text {e-ion }}$ is the Coulomb attraction between a polarized electron from an atomic Fe (in the liquid outer core) and another atomic Fe in the solid inner core. Note that the solid inner and liquid outer cores form a single system, and they are at a critical point due to liquid-solid coexistence. Furthermore, atomic $\mathrm{Fe}$ is highly polarized in liquidstate (at temperature, $\left.T_{1}\right)$ than in solid-state $\left(T_{2}\right)$ because $T_{1}>T_{2}$, regardless of its pressure. This Coulomb attraction,

$$
V_{\mathrm{Coulomb}}^{\text {e-ion }}=\frac{(-e)(+e)}{4 \pi \epsilon_{0}\left|\mathbf{R}_{\mathrm{Fe}}^{\text {solid }}-\mathbf{r}_{\mathrm{Fe}}^{\text {liquid }}\right|} \exp \left[-\mu r_{\mathrm{Fe}}^{\text {solid }}\right],
$$

where $\mathbf{R}_{\mathrm{Fe}}^{\text {solid }}$ and $\mathbf{r}_{\mathrm{Fe}}^{\text {liquid }}$ are the coordinates for atomic $\mathrm{Fe}$ in solid phase and a valence electron from an atomic $\mathrm{Fe}$ in liquid phase, respectively, $e$ is the electron charge, $\epsilon_{0}$ is the permittivity of free space, $\mu$ denotes the screening constant of proportionality, and $\exp \left[-\mu r_{\mathrm{Fe}}^{\text {liquid }}\right]$ captures the screening effect. In particular, the electron at $\mathbf{r}_{\mathrm{Fe}}^{\text {liquid }}$ is screened by the electron at $\mathbf{r}_{\mathrm{Fe}}^{\text {solid }}$ such that large screening effect corresponds to large $\mu$ that correctly gives small $V_{\text {Coulomb }}^{\text {e-ion }}$ and $V_{\text {Ramachandran }}$. Here, $V_{\text {Ramachandran is the complete version of the }}$ Ramachandran attraction between two nonbonded and neutral atoms due to asymmetric polarization. Whereas, $V_{\text {Ramachandran }}^{\mathrm{I}}$ is the mutual Ramachandran attraction due to symmetric polarization, also between two nonbonded neutral atoms. The required chemical reaction between two $\mathrm{Fe}$ atoms during cooling, occurs due to $V_{\text {Ramachandran }}$ (equation 5), while $V_{\text {Ramachandran (equation } 7 \text { ) is only }}^{\mathrm{1}}$ responsible for the attraction (stronger than the strongest vdW attraction) such that equation (7) cannot initiate any chemical reaction due to el-el repulsion. Therefore, we need to activate $V_{\text {Coulomb }}^{\text {e-ion }}$ (equation 6) to overcome the el-el repulsion, and consequently we obtain equation (5).

The second term on the right-hand side of equation (5) is the part that is responsible for a stronger attraction, stronger than the strongest standard vdW attraction (Arulsamy 2014a),

$$
V_{\text {Ramachandran }}^{\mathrm{I}}(R)=\frac{1 / \sqrt{2}-1}{R^{3 / 2}}\left[\frac{\hbar^{2} e^{2}}{2 m_{\mathrm{e}} \pi \epsilon_{0}}\right]^{1 / 2},
$$

where $m_{\mathrm{e}}$ and $\hbar$ denote the electron mass and Planck constant, respectively, $R$ is the distance between two interacting neutral Fe atoms, one from the solid, while the other from liquid outer core. These two Fe atoms are identical and therefore, we have the factor $1 / 2$ in equation (5) due to symmetric attraction. The asymmetric part is taken into account by equation (6). The Ramachandran interaction is guaranteed to be an attraction because $V_{\text {Ramachandran }}^{\mathrm{I}}(R)<0$ (due to $1 / \sqrt{2}-1<0$ ) and $V_{\text {Coulomb }}^{\text {e-ion }}<0$ (due to $-e$ ). Even though Ramachandran did not derive equation (7), he was the first to have discovered and defended (Ramakrishnan 2001; Balaram 2013; Vishveshwara 2014) the existence of a stronger attraction (stronger than the vdW type) between nonbonded neutral atoms in amino acids via numerical calculations (Ramachandran et al. 1963). Therefore, equation (7) (that was analytically derived earlier by introducing a large interaction constant between two neutral and polarizable atoms within the Drude model Hamiltonian), has been named after Ramachandran as it should be (Arulsamy 2014a).

Finally, in the above equations, we have physically removed (not by hand) the factor containing $\xi_{\text {liquid }}$ and $\xi_{\text {solid }}$, which are the energy-level spacings for atomic Fe in solid and liquid states, respectively (Arulsamy 2014b), because they are irrelevant in the present context for two reasons: (I) solid iron is a free-electron metal (meaning $\xi=0$ ), and (II) both liquid and solid phases composed of mostly iron atoms such that the atomic iron and impurity concentrations are constants, and consequently, $\xi$ is just a constant that can be taken to be zero.

We stress here that the Ramachandran attraction is not an ad hoc effect that can appear here or there by choice. In fact, it is one of the 
fundamental physical phenomena that applies to any interacting atoms under any condition where it reduces to the well-known van der Waals attraction if the atoms interact weakly, either due to inert atoms or large interatomic distance or both (Arulsamy 2014a). Hence, non-steady state at extreme conditions will not invalidate the theory of Ramachandran attraction where these conditions imply that the magnitude of this attraction is not a constant, but will vary such that different attractive strengths are expected on different regions of the Lehmann boundary, depending on temperature and pressure distributions. These varying attractive strengths will eventually determine the formation of hcp crystal-structure surfaces. This theoretical fact shall be captured and imposed as postulates in the subsequent section.

Obviously, we are not able to capture this time-dependent variation of the Ramachandran attraction and heat transport quantitatively in the presence of the said non-steady conditions. However, we can overcome this shortcoming by realizing that any attempt to evaluate the above variation quantitatively requires one to guess some arbitrary functions with adjustable parameters, which will not expose any new mechanism nor guarantee the correctness of the proposed postulates. Hence, our strategic move here is the right one - to build microscopic postulates to tackle iron solidification at the inner-core boundary such that its correctness and validity can be verified theoretically (on the basis of Ramachandran interaction theory), and with laboratory experiments. In these experiments, we just need to introduce different temperature and pressure distributions where the maximum temperature and pressure can be as low as $500 \mathrm{~K}$ and $1 \mathrm{~atm}$, respectively. Note that the physics of the Ramachandran interaction remains the same regardless of whether the critical point is at $5000 \mathrm{~K}$ $(300 \mathrm{GPa})$ or $500 \mathrm{~K}$ (1 atm).

\section{Results and discussion}

In the subsequent analysis, we shall address the difficult issues first - why P-wave travels faster along the polar path compared to an equatorial path, and also why $\mathrm{P}$-wave gets to pass through the western inner core hemisphere slowly in comparison to eastern hemisphere (see points (i) and (ii) at the end of introduction). Point (iii), which relies on straightforward arguments, comes last. Our strategy here is to first use the Ramachandran attraction to construct the relevant postulates to explain the outer core solidification with different atomic orientation. Subsequently, different atomic arrangements during solidification are shown to be responsible for changes in $\mathrm{P}$-wave velocity.

\subsection{Fluctuating super-rotation}

Solid inner core has been experimentally confirmed to rotate slightly faster than the mantle, about 0.3 to 0.5 degree $\mathrm{yr}^{-1}$ (Zhang et al. 2005), which amounts to $6-10 \mathrm{~km} \mathrm{yr}^{-1}$ or $0.2-0.3 \mathrm{~mm} \mathrm{~s}^{-1}$ by taking the radius of inner core, $r_{\mathrm{IC}}=1220 \mathrm{~km}$. However, further analysis points to the fact that this super-rotation does not continue indefinitely, but rather fluctuates with positive $\left(\Omega_{\mathrm{IC}}-\Omega_{\mathrm{OC}}>0\right)$ and negative $\left(\Omega_{\mathrm{IC}}-\Omega_{\mathrm{OC}}<0\right)$ inner core angular velocities (Tkalčić et al. 2013). Here $\Omega_{\mathrm{IC}}$ and $\Omega_{\mathrm{OC}}$ are the angular velocities for the inner and outer cores, respectively.

An hcp unit cell, which is a hexagonal primitive cell, contains four atoms on one of its smallest surface area $\left(S_{90}^{\text {hcp }}\right)$ per unit cell $\left(S_{90}^{\text {hcp }}=2 \times\right.$ $\left.(1 / 2) \times a_{\mathrm{L}} \times \sqrt{3} a_{\mathrm{L}} / 2\right)$ where $a_{\mathrm{L}}=2.1528 \AA$. See figure 1 for details. The same primitive cell contains two $(8 \times(1 / 8)+1)$ atoms per unit cell volume (not shown for clarity) where $c_{\mathrm{L}}=3.4351 \AA$, $\gamma=120^{\circ}, \alpha=90^{\circ}$, and $a_{\mathrm{L}}=b_{\mathrm{L}} \neq c_{\mathrm{L}}$ are the hcp-iron lattice parameters (Tateno et al. 2010) for temperature, $T=4820 \mathrm{~K}$ and pressure, $P_{r}=$ $332 \mathrm{GPa}$. Using these numbers, one can estimate the total number of atoms on inner core surface, which is found to be of the order of $10^{33}$, while the whole inner core contains atoms of the order of $10^{48}$ that eventually gives us the correct order for

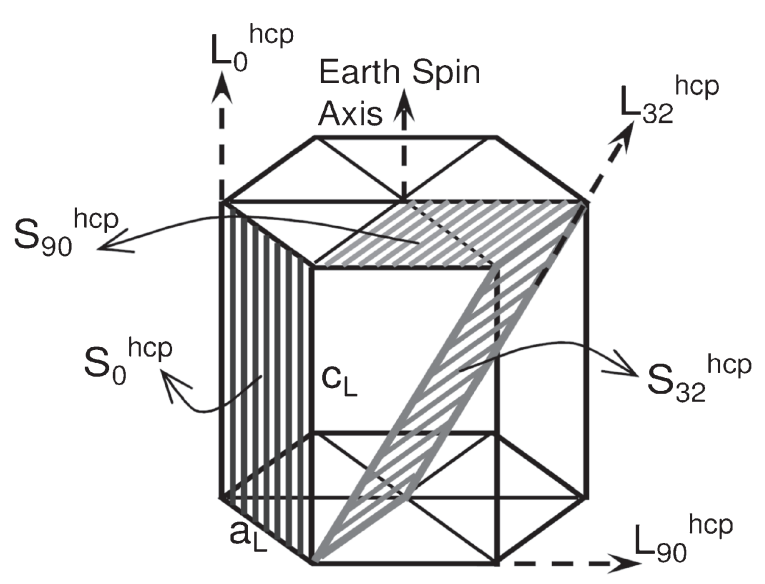

Figure 1. Hexagonal close-packed (hcp) crystal structure with $a_{\mathrm{L}}$ and $c_{\mathrm{L}}$ as lattice parameters. The unit cell of the hcp structure is the hexagonal primitive cell (one-third of the hcp structure) with two atoms per primitive unit cell volume. The Earth spin axis is drawn along the $c$-axis for convenience (not fixed, depends on the solidification mechanism). There are three main surfaces with the following definitions, $S_{90}^{\text {hcp }}\left(90^{\circ}\right.$ from $c$-axis $), S_{0}^{\text {hcp }}\left(0^{\circ}\right.$ from $c$-axis $)$ and $S_{32}^{\text {hcp }}\left(32^{\circ}\right.$ from $c$-axis). These surfaces, when divided by the number of surface atoms, give surface densities, whereas, $L_{90}^{\mathrm{hcp}}, L_{0}^{\mathrm{hcp}}$ and $L_{32}^{\text {hcp }}$ are called line densities (after dividing with the number of atoms between two points along the line). 
inner core mass $(M), 10^{22} \mathrm{~kg}$ (about $13 \mathrm{~g} \mathrm{~cm}^{-3}$ ) (Anderson 1989). Here, we have used Fe mass number, $55.85 \mathrm{~g} \mathrm{~mol}^{-1}$.

The numbers computed above rely on a series of crude and elementary calculations, which are also known as Fermi estimates (Nityananda 2014). These estimates are reliable and adequate for us to refocus our effort to understand the mechanism that can give rise to anisotropic inner-core solidification in the presence of fluctuating superrotation that causes the Ramachandran attraction to respond accordingly. In particular, the upper limit (Arunan 1999) for Ramachandran (or intermolecular) interaction strength is $50 \mathrm{kNm} \mathrm{mol}{ }^{-1}$ that gives a torque value of $10^{14} \mathrm{Nm}$ due to $V_{\text {Ramachandran acting on all inner core surface atoms }}$ $\left(10^{33}\right)$ by an equal number of atomic Fe from the liquid outer core. Here $10^{14} \mathrm{Nm}$ is insignificant to cause any changes to inner core rotation where the torque (Dumberry and Mound 2010) experienced by inner core is of the order of $10^{20} \gg$ $10^{14} \mathrm{Nm}$. This means that, the fluctuating superrotation is responsible for changing the strength of $V_{\text {Ramachandran }}$ accordingly, and not the other way round. Anyway, we now have sufficient information to construct the first postulate.

\subsubsection{Postulate 1}

If $\left|\Omega_{\mathrm{IC}}-\Omega_{\mathrm{OC}}\right|=\Delta \Omega \neq 0$ during super-rotation fluctuation, then $V_{\text {Ramachandran }}$ between Fe atoms is minimum. This minimum value can be further lowered to a new minimum if the temperature is high. Conversely, $V_{\text {Ramachandran }}$ is maximum if $\Delta \Omega \approx 0$, however, this maximum value is reduced to a lower maximum for high temperature.

Postulate 1 can be understood physically by noting that $V_{\text {Ramachandran }}$ is not maximally activated if inner core rotates faster or slower than outer core because the slight rotation stated above (ranges between 0.2 and $0.3 \mathrm{~mm} \mathrm{~s}^{-1}$ ) is very large in Ångström-scale or at the scale of an hcp unit cell where $0.3 \mathrm{~mm} \mathrm{~s}^{-1}=3 \times 10^{6} \AA \mathrm{s}^{-1}$. Apparently, the above angular velocities $\left(\Omega_{\mathrm{IC}}\right.$ and $\left.\Omega_{\mathrm{OC}}\right)$ should be in $\AA \mathrm{s}^{-1}$ because $V_{\text {Ramachandran }}$ refers to interaction between individual atoms at $\AA$-scale.

It is also a fact that temperature on inner core surface is not uniformly distributed giving rise to low and high temperature regions of inner core surface (Sumita and Olson 2002). This can lead to small and large mobilities (in $\AA \mathrm{s}^{-1}$ ) of atomic Fe from liquid outer core, respectively. Hence, high temperature reduces the strength of $V_{\text {Ramachandran, }}$ in which this relationship is only valid at critical points (when solid inner core coexists with liquid outer core) or during chemical reactions.
As a matter of fact, we cannot incorporate the inner-core rotation into equations (5-7) quantitatively; that is why we constructed Postulates 1 and 2 (see below) to claim that Ramachandran interaction is minimum when $\Delta \Omega$ is maximum, and vice versa. We shall invoke these postulates by-hand and use them together with equations (5-7) to address the seismic data analysis. In any case, the correctness of these postulates can be understood by realizing that the collision probability between the same Fe atoms (one-half of them from the solid phase, while the other half comes from the liquid outer core) is small if one of the phases (solid or liquid) is rotating faster or slower. If their rotations are identical $(\Delta \Omega \approx 0)$, then this probability (or the Ramachandran attraction) is maximum, giving rise to high-density atomic surface.

\subsection{Inner-core anisotropy: polar versus equatorial paths}

The temperature and pressure at the Lehmann boundary are about $6000 \mathrm{~K}$ and $300 \mathrm{GPa}$, and the only stable crystal structure at these magnitudes of $T$ and $P_{r}$ is the hexagonal close-packed, while face-centered cubic (fcc) is expected for much lower $T(<4000 \mathrm{~K})$ and $P_{r}(<100 \mathrm{GPa})$ where the melting point is nonlinearly proportional to pressure (Tateno et al. 2010; Anzellini et al. 2013). This additional experimental data, together with Postulate 1 leads us to construct Postulate 2.

\subsubsection{Postulate 2}

If $V_{\text {Ramachandran }}$ is small, for example in the presence of $\Delta \Omega \neq 0$ (from Postulate 1 ), then the atoms tend to arrange (via chemical reaction) to produce surface area with lowest atomic concentration or simply largest surface area per atom, $S_{32}^{\text {hcp }}=$ $\left(a_{\mathrm{L}} \times \sqrt{a_{\mathrm{L}}^{2}+c_{\mathrm{L}}^{2}}\right) / 4\left(32^{\circ}\right.$ from $c$-axis $)$, followed by a slightly smaller surface, $S_{0}^{\text {hcp }}=\left(a_{\mathrm{L}} \times c_{\mathrm{L}}\right) / 4\left(0^{\circ}\right.$ from $c$-axis) such that a normal vector through any one of these surfaces is also normal to inner core surface. For large $V_{\text {Ramachandran }}$ when $\Delta \Omega \approx 0$, atoms have high probability or tendency to form surface area with high atomic concentration or smallest surface area per atom, $S_{90}^{\mathrm{hcp}}=\left(\sqrt{3} a_{\mathrm{L}}^{2} / 2\right) / 4$ $\left(90^{\circ}\right.$ from $c$-axis) as the inner core surface where $S_{32}^{\text {hcp }}>S_{0}^{\text {hcp }}>S_{90}^{\text {hcp }}$.

Postulates 1 and 2 allow one to relate $\Delta \Omega \approx 0$ to large $V_{\text {Ramachandran, which then gives rise to }}$ atomic orientation with smallest surface area per atom, $S_{90}^{\text {hcp }}$ that forms the inner core surface. Since the fast axis for an hep crystal is normal to this surface, $S_{90}^{\text {hcp }}$, one can then readily surmise that 
super-rotation with minimum differential rotation $(\Delta \Omega \approx 0)$ leads to hcp crystal growth with its fast axis perpendicular to Earth spin axis. Apparently, the thickness of inner core surface with $S_{90}^{\text {hcp }}$ depends on the duration of $\Delta \Omega \approx 0$. This duration is denoted by the symbol, $t_{\Delta \Omega \approx 0}$. Different concentration of $S_{90}^{\text {hcp }}$ distributed in the western and eastern inner core hemispheres can lead to asymmetric $v_{\mathrm{P}}$ between those two hemispheres.

On the other hand, the condition, $\Delta \Omega \neq 0$ gives rise to the formation of largest surface areas per atom, $S_{32}^{\mathrm{hcp}}$ and $S_{0}^{\mathrm{hcp}}$, which are the dominant inner core surfaces. The two fast axes in this case are $32^{\circ}$ and $0^{\circ}$ from the Earth spin (or $c$ ) axis that can lead to the observed inner core cylindrical symmetry. Additionally, the thickness of inner core surface with $S_{32}^{\text {hcp }}$ and $S_{0}^{\text {hcp }}$ depend on the duration of $\Delta \Omega \neq 0$ where $t_{\Delta \Omega \neq 0}$ denotes the said duration. Obviously, fluctuating inner core super-rotation implies $t_{\Delta \Omega \approx 0}<t_{\Delta \Omega \neq 0}$ and consequently, the reported cylindrical symmetry is expected to be the dominant inner core symmetry with thicker $S_{32}^{\text {hcp }}$ and $S_{0}^{\text {hcp }}$ compared to $S_{90}^{\text {hcp }}$.

The $\mathrm{P}$-wave that passed through inner core should have propagated along different hcp surfaces, $S_{32}^{\text {hcp }}$ (about $32^{\circ}$ from $c$-axis), $S_{0}^{\text {hcp }}\left(0^{\circ}\right.$ from $c$ axis) and the smallest hcp surface $\left(90^{\circ}\right.$ from $c$-axis $)$, denoted by $S_{90}^{\text {hcp }}$. After putting all these together, equation (4) should read

$$
\left(v_{\mathrm{P}}\right)_{\theta}=\sqrt{c^{\mathrm{s}}}\left[x_{32} \frac{1}{\sqrt{\rho_{32}}}+y_{0} \frac{1}{\sqrt{\rho_{0}}}+z_{90} \frac{1}{\sqrt{\rho_{90}}}\right] \text {, }
$$

where $x_{32}, y_{0}$ and $z_{90}$ are the concentrations of their respective surfaces, $S_{32}^{\text {hcp }}, S_{0}^{\text {hcp }}$, and $S_{90}^{\text {hcp }}$ passed through by a particular $\mathrm{P}$-wave such that

$$
x_{32}+y_{0}+z_{90}=1 .
$$

In equation (4), we have used $i=1,2, \ldots$ but that notation turned out to be inconvenient in this specific case, and in the following analysis. Anyway, the relative sound velocities along these dominant hcp surfaces, $32^{\circ}, 0^{\circ}$ and $90^{\circ}$ can be predicted from equation (8). For example, unlike shear wave propagation, $\mathrm{P}$-wave travels through a three-dimensional crystal lattice in such a way that one can effectively separate the P-wave into one-dimensional components $(i, j, k)$ with only one dominant fast axis. This argument has been justified earlier in equation (4).

From equation (8), one should be clear about the conclusion: the axis along $L_{32}^{\mathrm{hcp}}$ is the fast axis, followed by $L_{0}^{\mathrm{hcp}}$, while the slowest axis is along $L_{90}^{\mathrm{hcp}}$. The analysis that has led to the above conclusion is not $a d$ hoc, as one can also correctly determine the fast axes for monatomic body-centered cubic (bcc) and face-centered cubic (fcc) crystal structures by using the line density technique defined earlier. For example, fast axis for monatomic bcc is diagonal, between points $(0,0,0)$ and $(1,1,1)$ through the body centered atom at $(1 / 2,1 / 2,1 / 2)$. Similarly, fcc's fast axis is also diagonally oriented, but only between two atoms at $(0,0,0)$ and $(1,1,1)$. We do not show such elementary calculations here. Of course, both bcc and fcc are assumed to have composed of mostly one type of atoms because the inner core itself is made of mostly iron atoms.

From $v_{\mathrm{P}}^{2} \propto 1 / \rho$ (see equation 8$)$, the axis with the lowest atomic line $\left(L^{\mathrm{hcp}}\right)$ and surface $\left(S^{\mathrm{hcp}}\right)$ densities is at $32^{\circ}$ from the hcp $c$-axis (see figure 1 ). In particular, $1 / \rho \propto L_{32}^{\mathrm{hcp}}=\left(\sqrt{a_{\mathrm{L}}^{2}+c_{\mathrm{L}}^{2}}\right) / 2=$ $2.03 \AA$ atom $^{-1}$ and $1 / \rho \propto S_{32}^{\mathrm{hcp}}=2.18 \AA^{2}$ atom $^{-1}$. For the other two surfaces, $L_{90}^{\mathrm{hcp}}=a_{\mathrm{L}} / 2=$ $1.08 \AA$ atom $^{-1}, S_{90}^{\mathrm{hcp}}=0.5 \AA^{2}$ atom $^{-1}, L_{0}^{\mathrm{hcp}}=$ $c_{\mathrm{L}} / 2=1.72 \AA \mathrm{atom}^{-1}$ and $S_{0}^{\mathrm{hcp}}=1.85 \AA^{2}$ atom $^{-1}$ where $L_{32}^{\mathrm{hcp}}>L_{0}^{\mathrm{hcp}}>L_{90}^{\mathrm{hcp}}$ and $S_{32}^{\mathrm{hcp}}>S_{0}^{\mathrm{hcp}}>S_{90}^{\mathrm{hcp}}$. The last two inequalities imply $\rho_{32}<\rho_{0}<\rho_{90}$ and therefore $\left(v_{\mathrm{P}}\right)_{32}>\left(v_{\mathrm{P}}\right)_{0}>\left(v_{\mathrm{P}}\right)_{90}$. Interestingly, our predicted inequality for $\left(v_{\mathrm{P}}\right)_{\theta}$ qualitatively agrees with experimental data of Mao et al. (1998) such that maximum $\left(v_{\mathrm{P}}\right)_{\theta}$ occurs for $0<\theta<90$ where $\theta_{\text {theory }}=32^{\circ}$, while $\theta_{\text {expt }}=45^{\circ}$. Note that the magnitudes of $\left(v_{\mathrm{P}}\right)_{\theta}$ are not predictable from our approach and calculating them is not our objective. What is important here is that our theory has got the maximum and minimum velocities registered in the radar, for example, the velocities are at least qualitatively in the right directions, which imply the basic physics discussed thus far is correct.

There are two equations (equations 8 and 9) and three unknowns, which imply we cannot solve these equations. But equation (8) can be used together with measured (Mao et al. 1998; Mattesini et al. 2013) polar path $\left(11.2409 \mathrm{~km} \mathrm{~s}^{-1}\right.$ ) and other (for $\theta=45^{\circ}, 0^{\circ}$ and $\left.90^{\circ}\right)$ velocities to obtain

$$
11.2409=11.8 x_{45}+11.1 y_{0}+11.0 z_{90} .
$$

Using equations (9 and 10), one can easily surmise that $y_{0} \approx z_{90}>x_{45}$, which is valid for polar paths (because $11.2409 \mathrm{~km} \mathrm{~s}^{-1}$ is the polar path velocity). The surmised inequality means that the concentrations of $S_{0}^{\text {hcp }}$ and $S_{90}^{\text {hcp }}$ are about equal, while the concentration of $S_{45}^{\mathrm{hcp}}$ is minimum, much smaller than $y_{0}$ and $z_{90}$. Thus, the probability for P-wave to pass through $S_{0}^{\mathrm{hcp}}$ or $S_{90}^{\mathrm{hcp}}$ is much larger than $S_{45}^{\mathrm{hcp}}$ surface. From our previous analysis using Postulate $2, \Delta \Omega \neq 0$ and $t_{\Delta \Omega \approx 0}<t_{\Delta \Omega \neq 0}$, we can easily rule out $S_{90}^{\text {hcp }}$ because it is an unlikely inner core surface to be formed during solidification for $\Delta \Omega \neq 0$. As a consequence, we are left with $S_{0}^{\text {hcp }}$ as the dominant surface along the polar path, and this surface is also responsible for inner core cylindrical symmetry. In summary, P-wave propagates faster along 
polar paths due to higher concentration of $S_{0}^{\text {hcp }}$ surface. This also means that $\mathrm{P}$-wave has to propagate slowly along the equatorial paths due to higher concentration of $S_{90}^{\text {hcp }}$ (perpendicular to inner core surface) due to the fact that $\left(v_{\mathrm{P}}\right)_{0}>\left(v_{\mathrm{P}}\right)_{90}$.

Our postulates listed above support the planar growth mechanism if the inner core rotates faster or slower than the liquid outer core. On the other hand, dendritic growth (Fearn et al. 1981; Shimizu et al. 2005) is a dominant mechanism when the differential rotation is small and negligible, giving rise to high-density surfaces. In reality both planar and dendritic growth mechanisms are responsible for the solidification of inner core. For example, the former is the dominant growth mechanism when the differential rotation is large $(\Delta \Omega \neq 0)$, while the latter is mostly responsible for $\Delta \Omega \approx 0$. However, we are only interested in the hcp crystal structure, its surface densities inside the inner core (just below the Lehmann boundary), not on the 'macroscopic' structure on the inner core surface. Apart from that, it is remarkable to note here that Postulate 2, though microscopic in principle, turns out to be not entirely new where the said postulate is also related to Bravais' law, which states that the crystal growth rate is the fastest for the greatest atomic density surface (Donnay and Harker 1937). In other words, a crystal prefers to grow along the direction normal to high density surface or with maximum $V_{\text {Ramachandran }}$. This means that, we can at least accept Postulate 2 as conceptually valid.

We now briefly point out why the available experimental data on solidification mechanism are not suitable to cross-check our postulates nor the seismic data analysis consistently and properly for three reasons. (i) The experiments were not properly set up, for example, there are no controlled fluid flow with differential rotation, (ii) the measurements were to capture the macroscopic solidification mechanism with respect to innercore macrostructure induced by the heat and mass transport via fluid flow, and (iii) the proposed models contain adjustable parameters such that the microscopic physics is not known (Deguen 2012). All three factors stated above have led to ambiguous conclusions extracted from the experiments. In particular, the so-called hydrodynamic interaction induced by the fluid flow onto the ice platelets have given rise to ice platelets with their $c$-axes aligned parallel to the fluid flow (ocean current) (Cherepanov 1971; Weeks and Gow 1978, 1980; Langhorne 1983; Langhorne and Robinson 1986). On the contrary, in another experimental set-up, the above hydrodynamic interaction due to fluid (salt water) flow 'within' the dendritic layer results in $c$-axis alignment perpendicular to the fluid flow (Bergman et al. 2002, 2003).
Added to that, the discussions of inner core solidification (Bergman et al. 2002, 2003) are ambiguous because there are actually two mutually perpendicular vectors that can be perpendicular to fluid flow, and we are not sure which one is referred to precisely on the basis of this relation, $a \circ b \circ c$, hcp crystal structure and crystal surfaces where ' $O$ ' abbreviates one of these, ' $=$ ', ' $<$ ' or ' $>$ '. Anyway, we shall briefly discuss (in the last section prior to conclusion) on how to set up a proper experiment to test the predictions and the seismic data on the basis of mineralogy and atomic scale physics.

\subsection{Asymmetric inner-core anisotropy}

As strange as it may seem, P-wave propagates (along the equatorial path) through the eastern hemisphere faster than the western hemisphere. There is a way to handle this problem without violating the fact that $\left(v_{\mathrm{P}}\right)_{\text {polar }}>\left(v_{\mathrm{P}}\right)_{\text {equator }}$. In Postulate 1 , we have justified that higher $T$ gives rise to high mobility (in $\AA \mathrm{s}^{-1}$ ) of iron atoms near the inner core surface, which is equivalent to activating this condition, $\Delta \Omega \neq 0$. As such, inner core regions with high and low temperatures activate these respective conditions, $\Delta \Omega \neq 0$ and $\Delta \Omega \approx 0$, and consequently, one can surmise that high $T$ or warmer inner core region (see figure 2) is mostly composed of $S_{0}^{\mathrm{hcp}}$, while the colder region has mostly $S_{90}^{\text {hcp }}$ as the inner core surface.

Hence, our prediction here is that the $\mathrm{P}$-wave propagates slower in an equatorial path through the warmer western inner core hemisphere (under the Atlantic Ocean and America) due to higher concentration of $S_{0}^{\text {hcp }}$ as the inner core surface. Whereas, the low-temperature eastern inner core hemisphere (under the Indian Ocean and Asia)

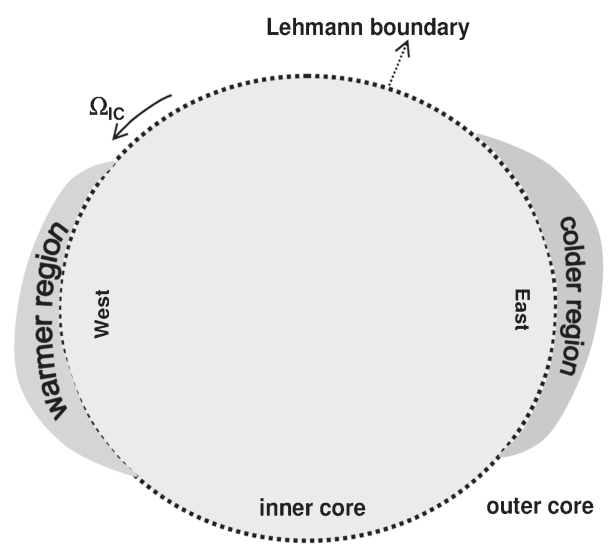

Figure 2. View from the north pole or top view of the inner core cross section with shaded warmer and colder regions. Inner-core rotation direction is denoted by $\Omega_{\mathrm{IC}}$ and the Lehmann boundary separates the solid inner core from the liquid outer core. Ramachandran interaction plays its part at this boundary. 
has relatively more $S_{90}^{\mathrm{hcp}}$ as the inner core surface (see figure 2) that eventually allows P-wave to travel faster via an equatorial path in the eastern hemisphere. Here, we have assumed that there are large enough differences in temperature and pressure such that there exist colder and warmer regions near the Lehmann boundary (as depicted in figure 2) due to large sizes of rotating inner and outer cores, which in turn allow some different lateral mass flow in the colder region compared to the warmer region. We are not sure about the magnitudes of the required differences in temperature and pressure that could induce the colder and warmer regions. This assumption may sound questionable (and we do not deny this), but our theoretical strategy is to first get the microscopic physics right (that can explain the observations consistently), and then make the macroscopic assumption(s) that can be fine-tuned. Doing it the other way round (getting the large-scale physics right, and then adjusting the Ångström-scale physics) will likely lead to contradicting conclusions and confusion.

\subsection{Depth-dependent P-wave attenuation and heat transfer}

One thing remains absolutely certain; solid iron (in whatever high pressure and temperature) is a textbook example of an excellent Fermi metal, readily obeying some of the well known free-electron concepts. In particular, both heat and charge are carried by electrons, thus heat transfer and electron flow can be discussed in a straightforward manner, even in the presence of external magnetic field and high frequency phonons. Given this background, $\mathrm{P}$-wave attenuation near the inner core surface, down to about $100 \mathrm{~km}$ in radial depth is somewhat expected because at this cross sectional depth of the inner core, the heat is close to melting point (with lower applied pressure) and therefore, the phonons have higher probability to be excited compared to much deeper part of the inner core. Here, the heat flow is from inner core surface to outer core to core-mantle boundary (Jacobs 1953; Nimmo and Alfe 2006; Nimmo 2007). The correctness of this heat-flow direction is unambiguous for these two reasons: (i) the inner core pressure is large such that it can withstand higher temperature without having to melt, and its temperature should be at least equal, if not higher than outer core, and (ii) inner core cooling is not sustainable (and physicologically false) if the heat were to flow from outer to inner core due to thermal energy accumulation at the center of inner core. Note that point (i) above does not imply that the temperature of inner core has to be higher than outer core, while point (ii) eliminates the possibility of low temperature inner core.

In this close-to-inner-core-surface region (distributed circularly around inner core), the electrons and phonons are in excited states such that the high frequency phonons interact strongly with excited and mobile electrons. This electron-phonon interaction actually retards the electrons mobility. The excitation probability for phonons along a low density path can be determined from the Bose-Einstein statistics and equation (4)

$$
f_{\mathrm{BES}}=\left[\exp \left(\frac{k \hbar}{k_{\mathrm{B}} T} \sqrt{\frac{c^{5}}{\rho}}\right)-1\right]^{-1},
$$

where $k_{\mathrm{B}}$ is the Boltzmann constant. As anticipated from equation (11), phonons have high probability to be excited along the low density path. In order to understand the low density path from equation (11), imagine two two-dimensional square lattice points, one with density, $\rho_{1}$ and interaction strength constant, $k_{1}$ and the second lattice points have $\rho_{2}$ and $k_{2}$. We now require this ratio, $k_{i} / \sqrt{\rho_{i}}$ to determine the low density path. For example, if $\rho_{2}>\rho_{1}$, then $k_{2}>k_{1}$ (from $\omega_{\mathrm{ph}}^{2} V \rho=k$ where $\omega_{\mathrm{ph}}^{2} V$ is a constant) and therefore, $k_{2} / \sqrt{\rho_{2}}>k_{1} / \sqrt{\rho_{1}}$, which eventually give the expected result $\left[f_{\mathrm{BES}}\right]_{1}>$ $\left[f_{\mathrm{BES}}\right]_{2}$ where the subscript 1 refers to the low density lattice points. Note that large $\omega_{\mathrm{ph}}$ value is required to increase the phase velocity (see equation 4), but high frequency phonons have lower probability to be activated by an earthquake (see equation 11).

The attenuation is smaller (from equation 11) in deeper regions of inner core due to constant$k$ effect; $k_{\text {deep }} / \sqrt{\rho_{\text {deep }}}<k_{\text {surface }} / \sqrt{\rho_{\text {surface }}}$ where $k_{\text {deep }} \approx k_{\text {surface }}$ and $\rho_{\text {deep }}>\rho_{\text {surface }}$. This inequality remains the same even if one has to assume that the temperature increases with inner core depth $\left((k / T \sqrt{\rho})_{\text {deep }}<(k / T \sqrt{\rho})_{\text {surface }}\right)$, which explains the observed attenuation near the inner core surface (Doornbos 1974; Souriau and Romanowicz 1996). See the discussion after equation (12) to understand why $k_{\text {deep }} \approx k_{\text {surface }}$. Moreover, the said anisotropy can be anisotropic as a result of asymmetric and anisotropic distribution of low line- and surface-densities as we have already discussed. We should also be aware here that $(k / T \sqrt{\rho})_{\text {deep }}<$ $(k / T \sqrt{\rho})_{\text {surface }}$ can only be true if the change in $T$, $\Delta T<\Delta k$, which implies, our theory requires small $\Delta T$ along the radial depth of inner core.

Warning: equations (4 and 11) are independently valid for a given density or line and surface densities defined earlier. In particular, if the density is changed by applying external pressure, then one needs a new set of equations (4 and 11) where the new equations also follow the same physics discussed above. For example, equation (4) for the 
new line or surface densities (of the same system) should read

$$
v_{\mathrm{P}}^{\text {new }}=\sqrt{c_{\text {new }}^{\mathrm{s}}} \sum_{i} \sqrt{\frac{1}{\rho_{i}^{\text {new }}}} .
$$

However, we cannot use equations (4 or 12) to evaluate the changes of $\mathrm{P}$-wave velocity with respect to density changes due to applied pressure (external disturbance) or other internal disturbances, namely, chemical doping. To examine such changes (between $v_{\mathrm{P}}$ and $v_{\mathrm{P}}^{\text {new }}$ ), one has to formulate the transformation from equation (4) to equation (12), which requires different physics than the one discussed using equations (4 and 12) individually for different applied pressure. In other words, we need to know how $c^{\mathrm{s}} \rightarrow c_{\text {new }}^{\mathrm{s}}$ when $\rho_{i} \rightarrow \rho_{i}^{\text {new }}$. Apart from the fact that the ionization energy theory (Arulsamy 2010) is useless for Fermi metals, it is also worth noting that in general the stated changes to $v_{\mathrm{P}}$ due to applied pressure cannot be evaluated with the energy level spacing renormalization group method (Arulsamy 2011) either because $\xi$ is found to be immune (or only weakly correlated) to changing density due to applied pressure, which has been proven for water at high pressure (Arulsamy et al. 2011).

In any case, Zhu Mao et al. (2012) and Antonangeli et al. (2004) have experimentally confirmed that $v_{\mathrm{P}} \propto \rho$ and $c^{\mathrm{s}} \propto \rho$, respectively, by means of changing applied pressure. However, the microscopic physics or mechanism responsible for such proportionalities (with respect to applied pressure) is not known, but from their experimental results $\left(v_{\mathrm{P}} \propto \rho\right.$ and $\left.c^{\mathrm{s}} \propto \rho\right)$, we can surmise that $\rho \propto \omega_{\mathrm{ph}}$, which is understandable if higher density is achieved via applied pressure. But such increase in $\rho$ (due to applied pressure) does not give rise to increasing interaction potential constant, $k$ significantly such that $k^{\text {new }} \approx k$ and therefore, $v_{\mathrm{P}}^{\text {new }}=\omega_{\mathrm{ph}}^{\text {new }} / k^{\text {new }}>$ $\omega_{\mathrm{ph}} / k=v_{\mathrm{P}}$. Here, $v_{\mathrm{P}}^{\text {new }}>v_{\mathrm{P}}$ is determined from changing applied pressure, and this $\mathrm{P}$-wave velocity inequality is in agreement with Zhu Mao et al. (2012) and Antonangeli et al. (2004). The technical reason for assuming $k^{\text {new }} \approx k$ comes from the fact that changing density due to applied pressure does not significantly affect the electron excitation probability or the energy level spacing $(\xi)$ (Arulsamy et al. 2011), which implies $\rho$ has negligible influence on $k$ if $\rho$ is determined from applied pressure.

\subsection{Falsification of Postulates 1 and 2}

Postulates 1 and 2 have been carefully constructed in such a way that one can rule them out with readily available experimental techniques without the need to solidify molten iron at extreme $T(\geq 5000$
$\mathrm{K})$ and $P_{r}(\geq 300 \mathrm{GPa})$. In particular, we just need to grow $\mathrm{Cu}_{1-x} \mathrm{Zn}_{x}$ crystal at a much lower temperature $(423 \mathrm{~K})$ and pressure (1 atm) (Kittel 1957) in the presence of rotating molten and solid phases with controlled differential rotation and temperature. Additionally, we require $x>0.9$ to obtain hcp $\mathrm{Cu}_{1-x} \mathrm{Zn}_{x}$ crystal $(c / a=1.86$ for $x=1)$. Such differential rotation can be implemented via a crystal growth method known as the Czochralski process (Czochralski 1918). Alternatively, the same process can be made to obey Postulates 1 and 2 in order to grow Co metal with hcp crystal structure. For example, high differential rotation $(\Delta \Omega \neq 0)$ growth has high probability to produce crystal with low density surface $\left(S_{32}^{\text {hcp }}\right.$ or $\left.S_{0}^{\text {hcp }}\right)$. This surface should be parallel to rotating crystal spin axis. On the contrary, zero or close to zero differential rotation $(\Delta \Omega \approx 0)$ will give rise to high density surface $\left(S_{90}^{\text {hcp }}\right)$.

\section{Additional notes}

Here, we further elaborate why and how Postulate 2 specifically refers to asymmetric and anisotropic cylindrical symmetry. In figure 3(a), the distribution of $S_{0}^{\text {hcp }}$ is spherically symmetric. This arrangement is definitely false even for an ideal inner core cooling because its linear velocity is always maximum at or near the equator, and the linear velocity decreases when we move towards the north or south pole. Postulate 2 does not support figure 3(a) in any way because the Ramachandran interaction is different by definition for different polar angles (the angle between the equator and one of the north or south pole) due to the changes in the linear velocity. Here, ideal solidification means that the inner core rotation and all other external parameters $\left(T, P_{r}, \nabla T, \nabla P_{r}\right.$, chemical composition and concentration along the Lehmann boundary) are all nonzero constants at the Lehmann boundary. The distribution of $S_{0}^{\mathrm{hcp}}$ in figure $3(\mathrm{~b})$ is true based on Postulate 2 (assuming again an ideal solidification of inner core for simplicity). In this ideal condition, the concentration of $S_{0}^{\text {hcp }}$ surface being parallel to inner core equator surface is large, and therefore, there exists a global north-south cylindrical anisotropy as depicted in figure 3(b). In nonideal conditions, the cross section in figure $3(\mathrm{~b})$ is still valid, but the three-dimensional distribution of $S_{0}^{\text {hcp }}$ is not so straightforward as already explained in our analysis earlier.

Earlier, we have said that our postulates support the mixing of planar and dendritic growth mechanisms depending on the inner core angular velocity. This statement precisely means that both dendritic and planar growths are responsible during the 

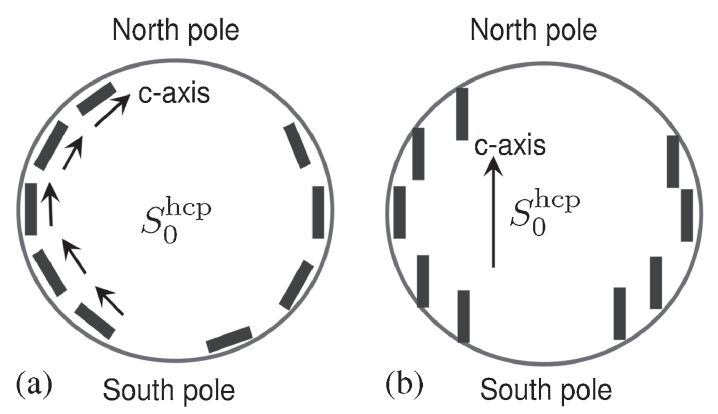

Figure 3. Earth's inner core cross sections from the north to south pole. The cross section shown in (a) indicates a spherically symmetric distribution of $S_{0}^{\text {hcp }}$. The one shown in (b) has the asymmetric and anisotropic cylindrical symmetry that can be derived from Postulate 2. The $c$-axis is mostly parallel to the north-south pole on the basis of Postulates 1 and 2 . The arrows in (a) indicate that the $c$-axis direction changes for different polar angles, while in (b) the $c$-axis is effectively (or globally) in one direction (along the north-south pole).

inner core cooling. It does not imply that these two mechanisms have to switch back and forth on the basis of inner core rotation. The formation and switching between different growth mechanisms are complex such that the switching is not a firstorder phase transition, and does not have a oneto-one correlation between the inner core superrotation fluctuation and the macroscopic growth mechanism. This means that, even if the experimental results of Bergman et al. $(2002,2003)$ and Cherepanov (1971) do not contradict with each other, their results are not unambiguously stated to be comparable. For example, it is simply stated that there is a so-called hydrodynamic interaction, which is different for different growth mechanisms without specifying the proper definition and correlation between the said interaction and growth processes.

\section{Conclusions}

We have attempted to tackle the one problem that has been side-stepped till now because it is difficult to obtain experimental verification under extreme conditions. The problem is associated to finding the solidification mechanism of molten iron alloy at the Lehmann (inner core) boundary in the presence of hot and cold inner core regions. Here, we have addressed this issue by constructing a first order theory with sufficient microscopic physics in such a way that one can readily rule out our postulates without the need to reach extreme conditions. Our strategy is to first devise an analytical technique on the basis of physical logic and inequalities to derive the one-dimensional $\mathrm{P}$-wave velocity representation that takes different atomic orientations and hcp unit cell surfaces into account. Subsequently, Ramachandran attraction is invoked to evaluate the possible solidification processes of molten iron at the Lehmann boundary.

The notion of fluctuating super-rotation is used together with Ramachandran interaction to find the most likely hcp crystal structure surfaces responsible for high and low $\mathrm{P}$-wave velocities by means of two constructed postulates. These postulates are used extensively to explain the following issues.

- The P-wave that travels along a polar path is faster than the wave that propagates along an equatorial path, which is due to Earth spin axis being parallel to high concentration of $S_{0}^{\mathrm{hcp}}$.

- Asymmetric anisotropy between the eastern and western hemispheres are due to the existence of warm and cold inner core regions.

- Anisotropic and depth dependent P-wave attenuation within the $100 \mathrm{~km}$ depth of inner core are shown to originate from constant- $k$ phonon excitation on the basis of Bose-Einstein statistics.

We have actually shown why and how the fluctuating inner core super-rotation turns out to be an important phenomenon that controls certain physical parameters at the Lehmann boundary and within the inner core itself. In particular, $\Delta \Omega \neq 0$ and $\Delta \Omega \approx 0$ directly determine these two processes: Fe-Fe Ramachandran interaction during outer core solidification and $\mathrm{Fe}-\mathrm{Fe}$ atomic orientation with different line and surface densities. These processes eventually explain the anisotropic and asymmetric hcp crystal orientation within inner core, namely, we now know why PKP wave travels at different velocities in different parts of inner core. For example, the said velocity is different along a polar path, compared to an equatorial path, and also, the equatorial paths in the eastern hemisphere have higher $\mathrm{P}$-wave velocities than in western hemisphere.

\section{Acknowledgements}

The author thanks Madam Sebastiammal Savarimuthu and Mr Arulsamy Innasimuthu, who have supported this work. Author is also grateful to the referees for the constructive criticism which helped to improve the paper remarkably.

\section{References}

Anderson D L 1989 Theory of the Earth; Blackwell Publications, Boston, USA.

Antonangeli D, Occelli F, Requardt H, Badro J, Fiquet G and Krisch M 2004 Elastic anisotropy in textured hcp-iron 
to $112 \mathrm{GPa}$ from sound wave propagation measurements; Earth Planet. Sci. Lett. 225 243-251.

Anzellini S, Dewaele A, Mezouar M, Loubeyre P and Morard G 2013 Melting of iron at Earth's inner core boundary based on fast X-ray diffraction; Science $\mathbf{3 4 0}$ 464-466.

Arulsamy A D 2010 Many-body Hamiltonian with screening parameter and ionization energy; Pramana J. Phys. $\mathbf{7 4}$ 615-631.

Arulsamy A D 2011 Renormalization group method based on the ionization energy theory; Ann. Phys. 326 541-565.

Arulsamy A D 2013 Nucleotide insertion initiated by van der Waals interaction during polymerase beta DNA replication; J. Chem. Sci. 125 1223-1235.

Arulsamy A D 2014a Chemical reaction due to stronger Ramachandran interaction; J. Chem. Sci. 126 677-689.

Arulsamy A D 2014b Quantum thermodynamics at critical points during melting and solidification processes; Indian J. Phys. 88 609-620.

Arulsamy A D, Kregar Z, Eleršič K, Modic M and Subramani U S 2011 Polarization induced water molecule dissociation below the first-order electronic-phase transition temperature; Phys. Chem. Chem. Phys. 13 15,17515,181 .

Arunan E 1999 Hydrogen bonding: A fascination forever!; Curr. Sci. 771233.

Arunan E 2013 Hydrogen bond seen, halogen bond defined and carbon bond proposed: Intermolecular bonding, a field that is maturing!; Curr. Sci. 105982.

Balaram P 2013 Remembering Ramachandran: Marking an anniversary; Curr. Sci. 104 155-156.

Bergman M I, Cole D and Jones J 2002 Preferred crystal orientations due to melt convection during directional solidification; J. Geophys. Res. 1072201.

Bergman M I, Agrawal S, Carter M S and MacleodSilberstein M 2003 Transverse solidification textures in hexagonal close-packed alloys; J. Crystal Growth 255 $204-211$.

Birch F 1964 Density and composition of the mantle and core; J. Geophys. Res. $694377-4388$.

Bullen K E 1985 An introduction to the theory of seismology; Cambridge Univ. Press, Cambridge, UK.

Cherepanov N 1971 Spatial arrangement of sea ice crystal structure; Probl. Arkt. Antarkt 38 176-181.

Creager K C 1997 Inner core rotation rate from small-scale heterogeneity and time-varying travel times; Science $\mathbf{2 7 8}$ 1284-1288.

Czochralski J 1918 Ein neues verfahren zur messung der kristallisationsgeschwindigkeit der metalle; Zeitschrift für Physikalische Chemie 92 219-221.

Deguen R 2012 Structure and dynamics of Earth's inner core; Earth Planet. Sci. Lett. 333-334 211-225.

Desiraju G R 2007 Chemistry: The middle kingdom; Resonance 12 44-60.

Donnay J D H and Harker D 1937 A new law of crystal morphology extending the law of Bravais; Am. Mineral. 22 446-467.

Doornbos D J 1974 The anelasticity of the inner core; Geophys. J. Roy. Astron. Soc. 38 397-415.

Dumberry M and Mound J 2010 Inner core-mantle gravitational locking and the super-rotation of the inner core; Geophys. J. Int. $181806-817$.

Emery A E H 1988 Pierre Louis Moreau de Maupertuis (1698-1759); J. Med. Genet. 25 561-564.

Fearn D R, Loper D E and Roberts P H 1981 Structure of the Earth's inner core; Nature 292 232-233.

Gutenberg B 1913 Über die konstitution des erdinnern, erschlossen aus erdbebenbeobachtungen; Z. Geophys. 14 $1217-1218$.
Gutenberg B and Richter C F 1936 Magnitude and energy of earthquakes; Science 83 183-185.

Hermansson K 2002 Blue-shifting hydrogen bonds; J. Phys. Chem. A 106 4695-4702.

Herndon J M 1992 Nuclear fission reactors as energy sources for the giant outer planets; Naturwissenschaften 79 7-14.

Hill R 1952 The elastic behavior of a crystalline aggregate; Proc. Phys. Soc. London 65 349-355.

Jacobs J A 1953 The Earth's inner core; Nature 172 297-298.

Jeffreys H 1926 The rigidity of the Earth's central core; Mon. Not. Roy. Astron. Soc. Geophys. Suppl. 1 371-383.

Kenneth B L N and Engdahl E R 1991 Travel times for global earthquake location and phase identification; Geophys. J. Int. 105 429-465.

Kittel C 1957 Introduction to Solid State Physics; John Wiley \& Sons, New York, USA.

Langhorne P 1983 Laboratory experiments on crystal orientation in $\mathrm{NaCl}$ ice; Ann. Glaciol. 4 163-169.

Langhorne P and Robinson W 1986 Alignment of crystals in sea ice due to fluid motion; Cold Regions Sci. Technol. 12 197-214.

Lehmann I 1936 Inner Earth; Bur. Cent. Seismol. Int. A 14 $3-31$.

Mani D and Arunan E 2013 The X-CY (X = O/F, Y= $\mathrm{O} / \mathrm{S} / \mathrm{F} / \mathrm{Cl} / \mathrm{Br} / \mathrm{N} / \mathrm{P}$ ) 'carbon bond' and hydrophobic interactions; Phys. Chem. Chem. Phys. 15 14,377-14,383.

Mao H K, Shu J, Shen G, Hemley R J, Li B and Singh A K 1998 Elasticity and rheology of iron above $220 \mathrm{GPa}$ and the nature of Earth's inner core; Nature 396 741-743.

Masters G and Gilbert F 1981 Structure of the inner core inferred from observations of its spheroidal shear modes; Geophys. Res. Lett. 8 569-571.

Mattesini M, Belonoshko A B, Tkalčić H, Buforn E, Udias A and Ahuja R 2013 Candy Wrapper for the Earth's inner core; Scientific Rep. 3 1-8.

McLeish A 1992 Geological Science; Thomas Nelson \& Sons, New York, USA.

Morelli A, Dziewonski A M and Woodhouse J H 1986 Anisotropy of the inner core inferred from PKIKP travel times; Geophys. Res. Lett. 13 1545-1548.

Nimmo F and Alfe D 2006. Properties and evolution of 1158 the Earths core and geodynamo; In: Advances in Science: Earth 1160 Science (eds) Sammonds P R and Thompson J M T, Imperial College Press, London, UK.

Nimmo F 2007 Energetics of the core; Treatise on Geophys. $831-65$.

Nityananda R 2014 Fermi and the art of estimation; Resonance $1973-81$.

Oldham R D 1906 Constitution of the interior of the Earth as revealed by earthquakes; Quart. J. Geol. Soc. 62 456-475.

Poupinet G, Pillet R and Souriau A 1983 Possible heterogeneity of the Earth's core deduced from PKIKP travel times; Nature 305 204-206.

Ramachandran G N, Sasisekharan V and Ramakrishnan C 1963 Stereochemistry of polypeptide chain configurations; J. Mol. Biol. 7 95-99.

Ramakrishnan C 2001 Ramachandran and his map; Resonance 6 48-56.

Rao K R 2002 Nuclear reactor at the core of the Earth! A solution to the riddles of relative abundances of helium isotopes and geomagnetic field variability; Curr. Sci. $\mathbf{8 2}$ 126-127.

Richards P G 2000 Earth's inner core - discoveries and conjectures; Astron. Geophys. 41 20-24.

Sankaran A V 2002 Recent concepts about heat source from the Earth's core; Curr. Sci. 83 932-934.

Shearer P M and Toy K M 1991 PKP(BC) versus PKP(DF) differential travel times and aspherical structure in the Earth's inner core; J. Geophys. Res. 96 2233-2247. 
Shimizu H, Poirier J P and Le Mouel J L 2005 On crystallization at the inner core boundary; Phys. Earth Planet. Inter. $15137-51$.

Song X D 1997 Anisotropy of the Earth's inner core; Rev. Geophys. 35 297-313.

Song X D 2000 Joint inversion for inner core rotation, inner core anisotropy, and mantle heterogeneity; J. Geophys. Res. 105 7931-7943.

Song X D and Helmberger D V 1993 Anisotropy of the Earth's inner core; Geophys. Res. Lett. 20 25912594.

Souriau A and Romanowicz B 1996 Anisotropy in inner core attenuation: A new type of data to constrain the nature of the solid core; Geophys. Res. Lett. 23 1-4.

Su W J and Dziewonski A M 1995 Inner core anisotropy in three dimensions; J. Geophys. Res. 100 9831-9852.

Sumita I and Olson P 2002 Rotating thermal convection experiments in a hemispherical shell with heterogeneous boundary heat flux: Implications for the Earth's core; J. Geophys. Res. 107 2169-2186.

Tanaka S and Hamaguchi H 1997 Degree one heterogeneity and hemispherical variations of anisotropy in the inner core from $\mathrm{PKP}(\mathrm{BC})-\mathrm{PKP}(\mathrm{DF})$ times; J. Geophys. Res. $1022925-2938$.
Tateno S, Hirose K, Ohishi Y and Tatsumi Y 2010 The structure of iron in Earth's inner core; Science 330 359-361.

Tkalčić H, Young M, Bodin T, Ngo S and Sambridge M 2013 The shuffling rotation of the Earth's inner core revealed by earthquake doublets; Nature Geosci. 6 497-502.

Tromp J 2001 Inner-core anisotropy and rotation; Ann. Rev. Earth Planet. Sci. 29 47-69.

Vishveshwara S 2014 Impact of theoretical chemistry on chemical and biological sciences; Resonance 19 347-367.

Weeks W and Gow A 1978 Preferred crystal orientations in the fast ice along the margins of the arctic ocean; J. Geophys. Res. 83 5105-5121.

Weeks W and Gow A 1980 Crystal alignments in the fast ice of Arctic Alaska; J. Geophys. Res. 85 1137-1146.

Woodhouse J H, Giardini D and Li X D 1986 Evidence for inner core anisotropy from splitting in free oscillation data; Geophys. Res. Lett. 13 1549-1552.

Zhang J, Song X D, Li Y, Richards P G, Sun X and Waldhauser F 2005 Inner core differential motion confirmed by earthquake waveform doublets; Science 309 1357-1360.

Zhu Mao, Lina J F, Liu J, Alatas A, Gao L, Zhao J and Mao H K 2012 Sound velocities of Fe and Fe-Si alloy, in the Earth's core; Proc. Natl. Acad. Sci. 109 10,239-10,244. 